ARTICLE

\title{
Durable protection against repeated penile exposures to simian-human immunodeficiency virus by broadly neutralizing antibodies
}

David A. Garber (10 ${ }^{1 凶}$, Debra R. Adams ${ }^{1}$, Patricia Guenthner ${ }^{1}$, James Mitchell', Kristen Kelley ${ }^{1}$, Till Schoofs ${ }^{2,6}$, Anna Gazumyan², Martha Nason ${ }^{3}$, Michael S. Seaman ${ }^{4}$, Janet McNicholl ${ }^{1}$, Michel C. Nussenzweig ${ }^{2,5}$ \& Walid Heneine ${ }^{1}$

Penile acquisition of HIV accounts for most infections among men globally. Nevertheless, candidate HIV interventions for men advance to clinical trials without preclinical efficacy data, due primarily to a paucity of relevant animal models of penile HIV infection. Using our recently developed macaque model, we show that a single subcutaneous administration of broadly neutralizing antibody (bNAb) 10-1074 conferred durable protection against repeated

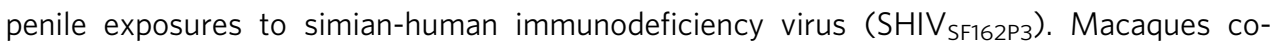
administered bNAbs 10-1074 and 3BNC117, or 3BNC117 alone, also exhibited significant protection against repeated vaginal SHIV $_{\text {AD8-EO }}$ exposures. Regression modeling estimated that individual plasma bNAb concentrations of $5 \mu \mathrm{g} \mathrm{ml} \mathrm{m}^{-1}$ correlated with $\geq 99.9 \%$ relative reduction in SHIV infection probability via penile (10-1074) or vaginal (10-1074 or 3BNC117) challenge routes. These results demonstrate that comparably large reductions in penile and vaginal SHIV infection risk among macaques were achieved at clinically relevant plasma bNAb concentrations and inform dose selection for the development of bNAbs as long-acting pre-exposure prophylaxis candidates for use by men and women.

\footnotetext{
${ }^{1}$ Laboratory Branch, Division of HIV/AIDS Prevention, Centers for Disease Control and Prevention, Atlanta, GA, USA. ${ }^{2}$ Laboratory of Molecular Immunology, The Rockefeller University, New York, NY, USA. ${ }^{3}$ Biostatistics Research Branch, National Institute of Allergy and Infectious Diseases, National Institutes of Health, Rockville, MD, USA. ${ }^{4}$ Center for Virology and Vaccine Research, Beth Israel Deaconess Medical Center, Harvard Medical School, Boston, MA, USA. ${ }^{5}$ Howard Hughes Medical Institute, The Rockefeller University, New York, NY, USA. ${ }^{6}$ Present address: GSK Vaccines, 1300 Wavre, Belgium. ${ }^{凶}$ email: david.garber@cdc.hhs.gov
} 
IV infection via the penis accounts for a majority of infections among the 17 million men estimated to be living with HIV globally, yet remains a relatively understudied route of virus transmission. It is the primary route of HIV acquisition among heterosexual men and is of relevance to MSM who practice insertive anal intercourse ${ }^{1}$. Current strategies to reduce one's risk of acquiring HIV infection via the penis include condom usage, male circumcision and taking antiretroviral drugs for preexposure prophylaxis (PrEP $)^{2-5}$. Regrettably, each of these prevention strategies has limitations that curb effectiveness. For example, even when used consistently, condoms are $<80 \%$ effective in reducing HIV incidence among uninfected partners of HIV-discordant heterosexual couples ${ }^{2}$. Similarly, male circumcision, in which all or part of the penile foreskin is removed surgically, confers incomplete protection against HIV-reducing infection risk by $50-60 \%{ }^{5}$. With regard to PrEP, once-daily oral tenofovir disoproxil fumarate (TDF) or TDF-emtricitabine (FTC) combination reduced HIV infection risk by $63 \%$ or $84 \%$, respectively, among heterosexual men with a known HIVinfected partner ${ }^{3}$. Among MSM, clinical trials have shown daily oral TDF-FTC to reduce overall HIV incidence either by $44 \%$ (with significantly greater reductions (92-95\%) among trial participants who had detectable study drugs in plasma) $)^{6}$, or by $86 \%$ (PROUD Study) ${ }^{7}$. In addition, on-demand dosing with TDF-FTC, rather than daily dosing, demonstrated an $86 \%$ reduction in HIV incidence as compared with placebo controls (IPERGAY Study) $^{8}$. While oral TDF-FTC can be highly efficacious in preventing HIV infection, users' lack of adherence to drug regimens is a major behavioral impediment that limits PrEP effectiveness 9 .

As a result, development of long-acting HIV intervention products that require relatively less frequent dosing remains a top priority in the HIV prevention and treatment fields. Passive immunization using recombinant monoclonal antibodies capable of neutralizing diverse HIV isolates (broadly neutralizing antibodies (bNAbs)) is a promising approach to achieve longacting HIV prevention. The Antibody-Mediated Prevention (AMP) Study currently is evaluating bNAb VRC01, when administered via intravenous infusion once every 8 weeks, for safety and efficacy to prevent HIV infection among high-risk women (HVTN 703/HPTN 081; NCT02568215) and among men and transgender persons who have sex with men (HVTN 704/HPTN 085; NCT02716675) ${ }^{10}$. However, because HIV transmission among MSM occurs more commonly via the rectal than the penile route $e^{1,11}$, the AMP Study is not poised specifically to evaluate antibody-mediated protection against penile HIV acquisition.

Many other bNAbs, which exhibit greater potency or neutralization breadth than VRC01 are being developed and are in clinical evaluation ${ }^{12-14}$. Some of the most clinically advanced among these are 10-1074, which targets the base of the third variable loop and surrounding glycans on the HIV envelope protein (Env), and 3BNC117, which targets the CD4 binding site ${ }^{15,16}$. Both have been shown to be safe and well tolerated in HIV-infected and uninfected individuals, and capable of suppressing HIV viremia in viremic subjects ${ }^{12,17-20}$.

During preclinical development, HIV prevention products or vaccines often are evaluated for protective efficacy in nonhuman primate models. These infection models are predicated upon exposing macaques to a single high-dose, or repeated low-doses of simian-human immunodeficiency virus (SHIV), most commonly via rectal or vaginal routes ${ }^{21,22}$. bNAbs $10-1074$ and 3BNC117 have been shown to protect macaques against rectal infection with $\mathrm{SHIV}_{\mathrm{AD} 8-\mathrm{EO}^{23,24}}$. However, their efficacy in preventing infection via the other major mucosal routes of HIV infection-namely penile or vaginal, has not been described. Nor is it known how these bNAbs' correlates of protection compare among all mucosal infection routes.

In the absence of penile animal model data, it is difficult to determine how efficacious PrEP regimens are in preventing HIV infection across the penile mucosa during insertive intercourse (vaginal or rectal) with an infected individual. Myriad differences exist between penile, vaginal, and rectal mucosa that may modulate HIV susceptibility or PrEP pharmacokinetics. These include differences in epithelial thickness or composition, presence or absence of mucus, mechanical trauma incurred via sexual intercourse, or prevalent microbiota ${ }^{25-32}$. Despite the public health relevance of penile HIV infection, preclinical studies of the efficacy of PrEP or other biomedical preventions against this route are limited, due largely to a lack of relevant animal models. This represents a knowledge gap with unknown impact on current and future translational efforts to develop new candidate HIV interventions for men.

Recently, we developed a repeat-exposure penile SHIV infection model in rhesus macaques to evaluate the protective efficacy of biomedical preventions against HIV $^{33}$. The model is predicated upon repeatedly exposing multiple penile tissues that are relevant for HIV acquisition in humans, including the inner foreskin, glans, and distal urethra, to limiting doses of $\mathrm{SHIV}_{\mathrm{SF} 162 \mathrm{P} 3}$ (i.e., doses that do not result in systemic infection among all animals following a single challenge) using a nontraumatic technique that precludes inadvertent perturbation of the penile epithelium ${ }^{33}$. Here, we utilized this penile infection model to assess the protection conferred by passive bNAb immunization. We chose to evaluate 10-1074, which exhibits potent neutralization of $\mathrm{SHIV}_{\mathrm{SF162P3}}$, and show that a single subcutaneous dose of 10-1074 durably protected macaques against repeated penile $\mathrm{SHIV}_{\mathrm{SF} 162 \mathrm{P} 3}$ challenges.

Because the efficacy of 10-1074 against vaginal challenge had not been evaluated, we also tested its protective efficacy in a repeat low dose challenge model. However, the greatest burden of HIV infection of women is in sub-Saharan Africa, where multiple HIV1 subtypes predominate ${ }^{34}$. In vivo, in vitro, and in silico modeling predicts that bNAbs used in combination will achieve greater neutralization coverage ${ }^{35-40}$. To this end, several phase-I studies evaluating bNAb combinations are ongoing ${ }^{14}$, and one that evaluated administering $10-1074$ in combination with $3 \mathrm{BNC} 117$ recently has been reported ${ }^{20}$. Therefore, we elected to evaluate 10-1074 in combination with $3 \mathrm{BNC117}$. Because $\mathrm{SHIV}_{\mathrm{SF} 162 \mathrm{P} 3}$ is not sensitive to neutralization by $3 \mathrm{BNC117}$, we chose to challenge vaginally with $\mathrm{SHIV}_{\mathrm{AD} \text {-EO, which is sensitive to both } 3 \mathrm{BNC117}}$ and 10-1074. As such, our penile and vaginal challenge models used SHIVs that are similarly susceptible to neutralization by 10-1074, with $\mathrm{IC}_{80}$ values of 0.20 or $0.25 \mu \mathrm{g} \mathrm{ml}^{-1}$ against the replication competent challenge virus stock of $\mathrm{SHIV}_{\mathrm{SF} 162 \mathrm{P} 3}$ or SHIV $_{\text {AD8-EO, respectively. We show that subcutaneously delivered }}$ 10-1074 and 3BNC117 exhibited different pharmacokinetics including a longer plasma persistence of 10-1074 than 3BNC117, providing an opportunity to measure efficacy against vaginal challenge of the bNAb combination and of 10-1074 alone. We used probit regression analyses to compare plasma bNAb concentrations that reduced penile or vaginal infection.

\section{Results}

Protection by 10-1074 against repeated penile SHIV challenges. We sought to determine the protective efficacy that passive immunization with 10-1074 conferred against SHIV acquisition by the penile route in rhesus macaques. Six macaques in the treatment group (Group-1) received a single subcutaneous injection of $10-1074$ (human $\mathrm{IgG}_{1}$ isotype; does not contain the LS or any other half-life modifying mutations) at $10 \mathrm{mg}$ per $\mathrm{kg}$ body weight at 1 week prior to the start of SHIV challenges 
a bNAb
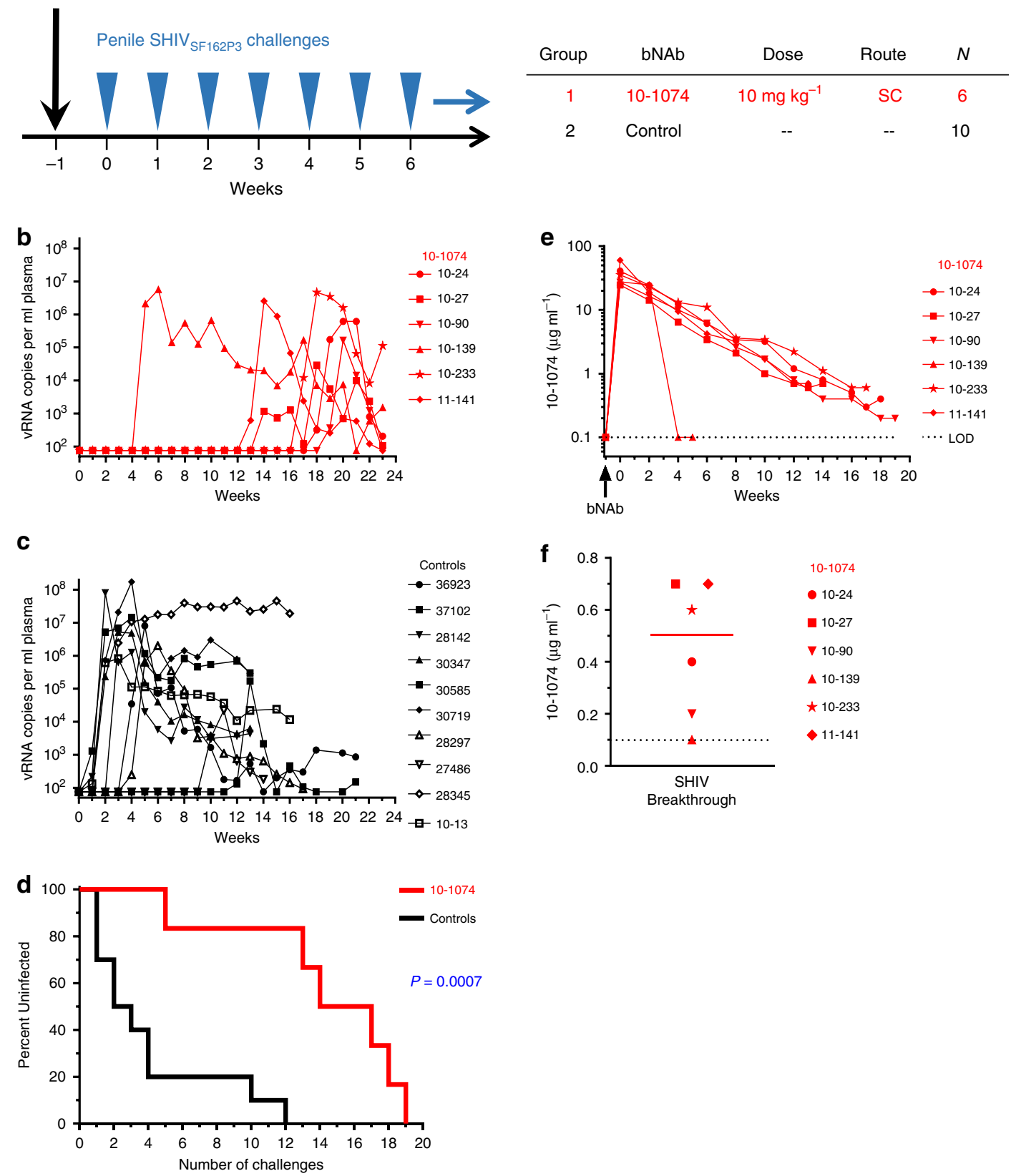

Fig. 1 Passive immunization of macaques with bNAb 10-1074 delays SHIV acquisition following repeated penile SHIV challenges. a Study design to assess the protective efficacy of 10-1074 against repeated penile SHIV challenges. Rhesus macaques $(N=6$; Indian origin) were injected subcutaneously once with 10-1074 $\left(10 \mathrm{mg} \mathrm{kg}^{-1}\right)$. Beginning 1 week later, macaques were challenged repeatedly, once per week, via the penis with $\mathrm{SHIV}_{\mathrm{SF} 162 \mathrm{P} 3}\left(200 \mathrm{TCID}_{50}\right.$ into the prepuce pouch and $16 \mathrm{TCID}_{50}$ into the distal urethra) until systemic SHIV infection was confirmed via plasma viral load assay. Control macaques $(N=10)$ received no antibody, but were challenged identically. Plasma viral load (vRNA copies $\mathrm{ml}^{-1}$ ) determined in 10-1074-treated macaques $(\mathbf{b})$ or untreated controls (c). Symbols denote individual animals. $\mathbf{d}$ Percentages of macaques remaining uninfected at 7 days following administration of the indicated cumulative number of SHIV challenges. Statistical difference between groups was measured using a two-sided log-rank test $(P=0.0007)$. e Plasma levels of 10-1074 ( $\mu \mathrm{g} \mathrm{ml}^{-1}$ ) were determined via TZM-bl neutralization assays using 10-1074-sensitive pseudovirus X2088.c9. f Plasma concentrations of 10-1074 in individual macaques $(N=6)$ at the time of SHIV breakthrough (values from e). The solid line denotes the group median; the dotted line denotes the limit of detection for 10-1074. Source data are provided in Supplementary Tables or a Source data file. SHIV infection data for animals in the control group have been reported previously in a paper describing the development of our penile SHIV infection model ${ }^{33}$ and are reproduced here to facilitate comparison between the treatment and control groups.

(Fig. 1a). We performed nontraumatic penile challenges with SHIV using a 'no contact' technique that eliminated inadvertent perturbation of the penile mucosa during the inoculation procedure itself, while simultaneously exposing multiple susceptible penile tissues to the challenge virus ${ }^{33}$. For each virus challenge, macaques received $200 \mathrm{TCID}_{50} \mathrm{SHIV}_{\mathrm{SF}_{162} \mathrm{P} 3}$ into the prepuce pouch and $16 \mathrm{TCID}_{50} \mathrm{SHIV}_{\text {SF162P3 }}$ into the distal urethra. Ten historical control animals (Group-2) had received no antibodies and had been challenged identically with the same stock of SHIV $_{\text {SF162P3 }}$ (Fig. 1a). Penile SHIV $_{\text {SF162P3 }}$ challenges were 
repeated once weekly until systemic infections were confirmed via positive RT-qPCR plasma viral load assay for macaques in the treatment group (Fig. 1b) or control group (Fig. 1c). Macaques that received a single injection of 10-1074 became infected following a median of 15.5 weekly challenges (range $=5-19$ ) and were protected significantly longer than untreated control animals, which became infected following a median of 2.5 challenges (range $=1-12)(P=0.0007$, Log-Rank test $)$ (Fig. 1d). Of note, the earliest infection among macaques administered 10-1074 occurred in animal 10-139 following five $\mathrm{SHIV}_{\mathrm{SF} 162 \mathrm{P} 3}$ challenges and corresponded with an abrupt decline in the neutralization activity of 10-1074 in plasma between 3 and 5 weeks post injection, due to the development of an antidrug antibody (ADA) response (Fig. 1b, Supplementary Fig. 1) ${ }^{41,42}$. Protection among 10-1074treated macaques that did not exhibit such ADA responses was relatively greater, showing median protection against 17 weekly challenges (range $=13-19)$. No differences in levels of peak viremia, or area under the curve (AUC) through 7 weeks post infection were observed between 10-1074-treated and control groups (Supplementary Fig. 1).

Plasma levels of 10-1074 exhibited a mean maximum concentration $\left(C_{\max }\right)$ of $36.0 \pm 13.3 \mu \mathrm{g} \mathrm{ml}^{-1}$ at 7 days following antibody injection, which was the earliest postinjection timepoint assayed (Fig. 1e). Plasma levels of 10-1074 exhibited an average half-life of $15.5 \pm 4.0$ days and remained above the level of detection $\left(0.10 \mu \mathrm{g} \mathrm{ml}^{-1}\right)$ for $15-20$ weeks following injection in 5 of 6 treated animals (Fig. 1e; (Supplementary Table 1)). At the time of SHIV breakthrough (i.e., first detectable plasma viremia), the median plasma concentration of 10-1074 among all treated macaques was $0.50 \mu \mathrm{g} \mathrm{ml}^{-1}$ (range: $<0.10-0.70 \mu \mathrm{g} \mathrm{ml}^{-1}$ ) (Fig. 1f). Excluding the animal that developed an early ADA response against 10-1074 and had an undetectable level of 10-1074 at the time of SHIV breakthrough, the median plasma concentration of $10-1074$ at the time of SHIV breakthrough was $0.60 \mu \mathrm{g} \mathrm{ml}^{-1}$ (range of $0.20-0.70 \mu \mathrm{g} \mathrm{ml}^{-1}$ ).

To evaluate antibody-mediated protection against penile infection, we used probit regression to estimate the per-challenge probability of SHIV infection as a function of imputed plasma 10-1074 levels (Fig. 2), excluding animal 10-139 who exhibited an early ADA response. Among no-antibody controls, each SHIV challenge presented a 0.25 chance of infection (Fig. 2; 95\% CI 0.13-0.41). The probit model estimated $>100-, 9.5-$, and 1.6-fold reductions of this infection probability to $<0.001,0.002$, or 0.093 for plasma $10-1074$ concentrations of $5.0,3.0$, or $1.0 \mu \mathrm{g} \mathrm{ml}^{-1}$, respectively. Bootstrap fitting of the probit regression model estimated a 0.01 probability of infection following a single penile SHIV challenge at a plasma $10-1074$ concentration of $2.28 \mu \mathrm{g} \mathrm{ml}^{-1}$ (95\% CI: $1.27,3.47$ $\left.\mu \mathrm{g} \mathrm{ml}^{-1}\right)$.

Protection by bNAbs against repeated vaginal SHIV challenges. bNAbs 10-1074 and 3BNC117 are among the most clinically advanced second generation bNAbs and have not been evaluated previously in nonhuman primate models for protection against vaginal SHIV challenge. Because bNAbs targeting nonoverlapping epitopes on HIV Env could be used in combination to achieve additive neutralization activity or increased breadth of neutralization ${ }^{35-40}$, we evaluated protection against repeated vaginal SHIV challenges among macaques that received a combination of 10-1074 and 3BNC117, or 3BNC117 singly.

Macaques in the treatment groups (Group-1, -2) received a onetime subcutaneous injection of both $10-1074$ and 3BNC117 (10 mg each $\mathrm{kg}^{-1}$, Group-1) or 3BNC117 singly (10 mg kg-1, Group-2) 1 week prior to the start of vaginal $\mathrm{SHIV}_{\mathrm{AD} 8-\mathrm{EO}}$ challenges. Both bNAbs were of human $\operatorname{IgG}_{1}$ isotype and did not contain the LS or any other half-life modifying mutations. Macaques in the control

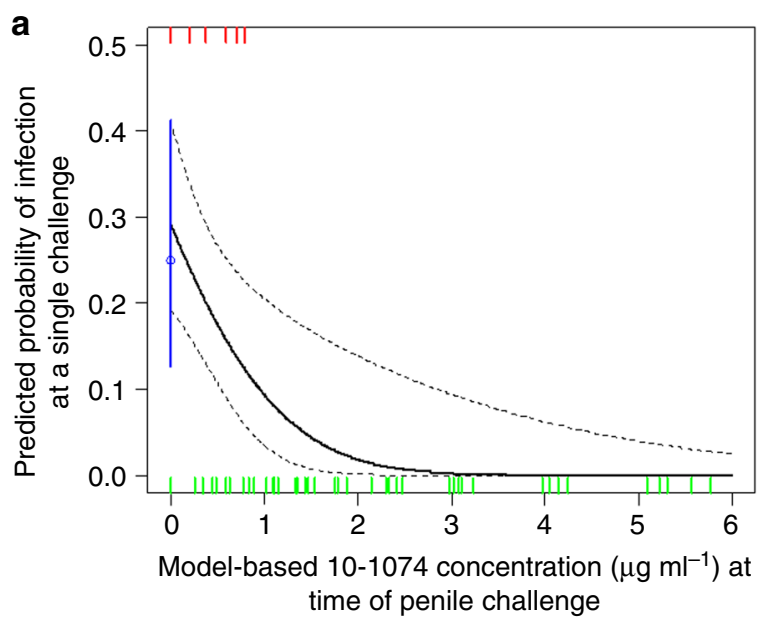

b

\begin{tabular}{ccc}
$\begin{array}{c}10-1074 \\
\left(\mu \mathrm{gl}^{-1}\right)\end{array}$ & $\begin{array}{c}\text { Infection probability per } \\
\text { penile SHIV challenge }\end{array}$ & $\begin{array}{c}\text { Estimated relative reduction } \\
\text { of infection probability } \\
\text { compared to controls }\end{array}$ \\
\hline 5.0 & $<0.001$ & $>0.99$ \\
3.0 & 0.002 & 0.99 \\
1.0 & 0.093 & 0.63
\end{tabular}

Fig. 2 10-1074 antibody concentration predicts the probability of infection following penile SHIV $\mathbf{S F 1 6 2 P 3}_{\mathbf{3}}$ challenge. a Probit regression analysis was used to estimate the per-challenge probability of penile SHIV acquisition as a function of imputed 10-1074 concentration in plasma. The per-challenge probability of infection among untreated control animals was 0.25 (95\% Cl 0.13-0.41) and is denoted in blue. Tick marks represent SHIV challenge events that either did (red) or did not (green) result in systemic infection. The solid black line is the model-based prediction; dotted black lines depict $90 \%$ pointwise confidence intervals. b Model-based estimates of SHIV infection probabilities following a single penile challenge and reduction of infection probability as compared with controls, at the indicated plasma concentrations of 10-1074.

group (Group-3) were not administered any antibodies and were challenged identically with $\mathrm{SHIV}_{\mathrm{AD} 8-\mathrm{EO}}$ (Fig. 3a). Macaques in all groups received intramuscular injections of depot medroxyprogesterone acetate (DMPA; $30 \mathrm{mg}) 2$ weeks prior to the first SHIV challenge (corresponding to 1 week prior to bNAb administration in the treatment groups), and every 4 weeks thereafter, to normalize vaginal SHIV susceptibility via progestin-mediated thinning of the

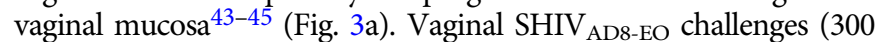
$\mathrm{TCID}_{50}$ ) were repeated once weekly until systemic infections were confirmed via positive RT-qPCR viral load assay for macaques in the combination treatment group (Fig. 3b), 3BNC117-only group (Fig. 3c), or untreated control group (Fig. 3d).

Macaques that received a single injection of only 3BNC117 showed breakthrough SHIV infections following a median of five challenges (range $=4-7$ ) and were protected significantly longer than were untreated controls, which became infected following a median of two SHIV challenges (range = $1-3)(P=0.0018$, Log-Rank test; Fig. 3e). More importantly, macaques in the $10-1074+3 \mathrm{BNC} 117$ combination group were protected against a median of 11 weekly challenges (range $=$ 10-12), which was significantly greater than that conferred following administration of 3BNC117 alone $(P=0.0012$, Logrank test) or observed among untreated controls $(P=0.0042$, Log-Rank test) (Fig. 3e). Levels of peak viremia and early SHIV replication, measured as vRNA AUC through 7 weeks post infection, were similar among all groups (Supplementary Fig. 3). 
a

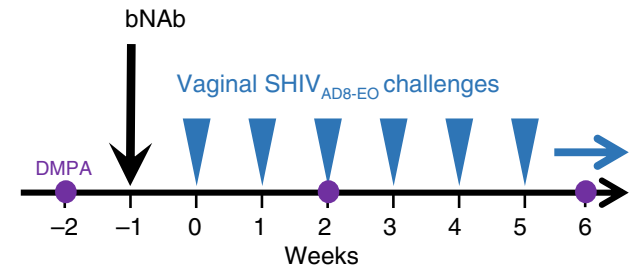

\begin{tabular}{ccccc} 
Group & bNAb & Dose & Route & $N$ \\
\hline 1 & $\begin{array}{c}10-1074+ \\
\text { 3BNC117 }\end{array}$ & $10 \mathrm{mg} \mathrm{ea}_{\mathrm{kg}} \mathrm{kg}^{-1}$ & SC & 5 \\
2 & 3BNC117 & $10 \mathrm{mg} \mathrm{kg}^{-1}$ & SC & 6 \\
3 & Control & -- & -- & 3
\end{tabular}

b
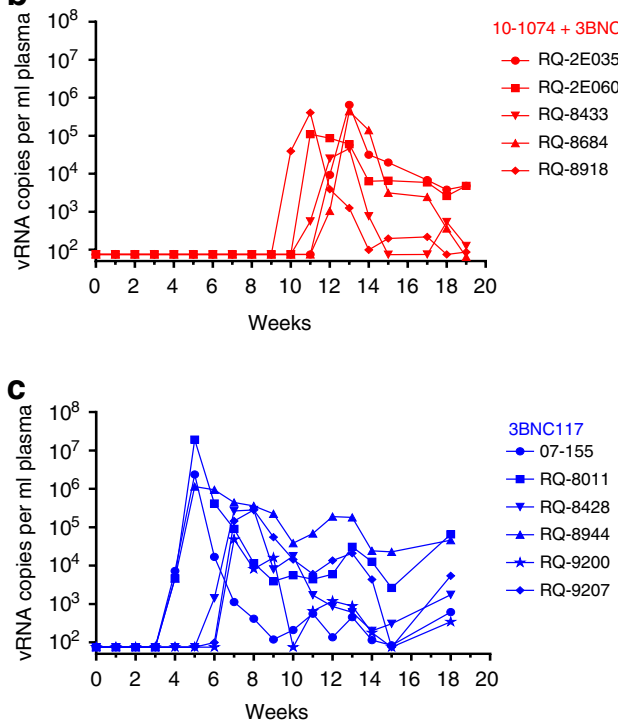

d

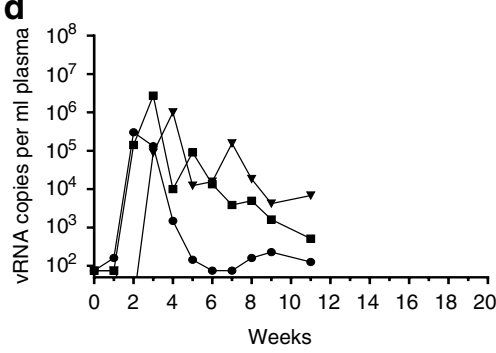

Controls

- RQ-8841

- RQ-8910

- RQ-9098
10-1074 + 3BNC117

- RQ-2E035

$\rightarrow$ RQ-2E060

$\rightarrow$ RQ-8433

$\rightarrow \mathrm{RQ}-8684$

$\rightarrow$ RQ-8918

\section{f}

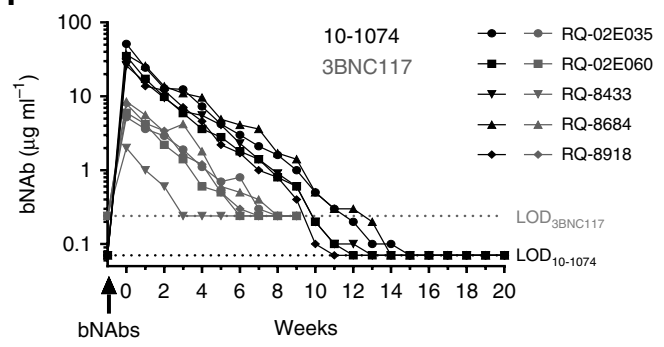

g

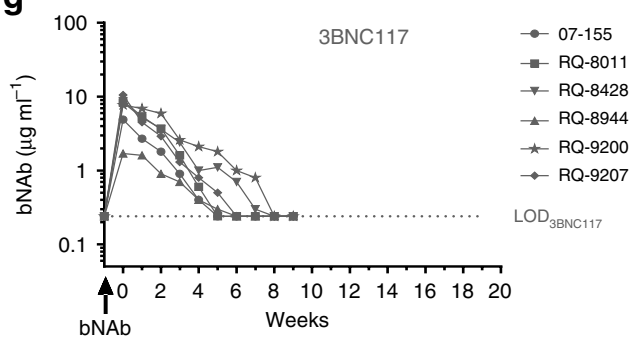

h
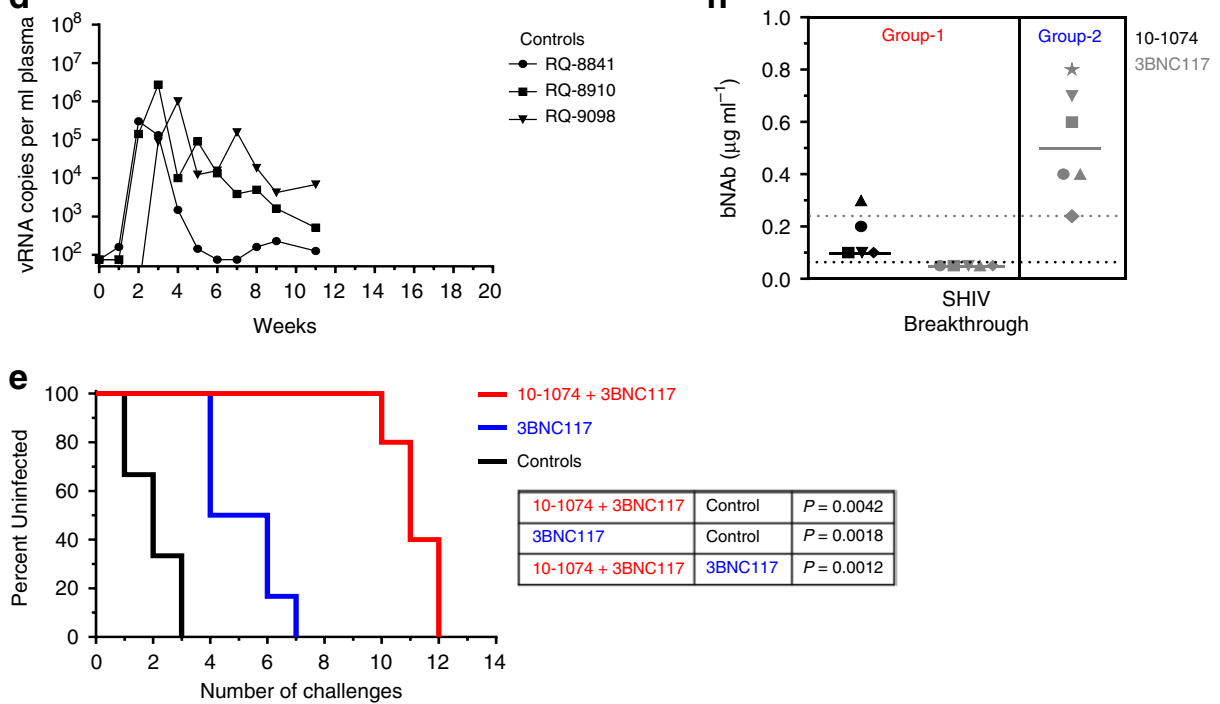

Fig. 3 Passive immunization with bNAbs 10-1074 and 3BNC117 in combination, or 3BNC117 singly, delays SHIV acquisition following repeated vaginal challenges in DMPA-treated macaques. a Rhesus macaques (Chinese origin) were injected subcutaneously once with a combination of bNAbs 10-1074 and 3BNC117 (10 mg each kg-1; $N=5)$ or 3BNC117 singly $\left(10 \mathrm{mg} \mathrm{kg}^{-1} ; \mathrm{N}=6\right)$. Commencing 1 week later, macaques were challenged repeatedly, once per week, intravaginally with $\mathrm{SHIV}_{\mathrm{AD} 8-\mathrm{EO}}\left(300 \mathrm{TCI} \mathrm{D}_{50}\right)$ until systemic SHIV infection was confirmed via plasma viral load assay. Control macaques $(\mathrm{N}=3)$ received no antibody but were challenged identically. Animals in both groups received DMPA (30 mg) intramuscularly at 2 weeks before the first SHIV challenge and every 4 weeks thereafter to normalize SHIV susceptibility. Plasma viral loads (vRNA copies $\mathrm{ml}^{-1}$ ) for macaques in the combination bNAb group (b), single bNAb group (c) or untreated control group (d). Symbols denote individual animals. e Percentages of macaques remaining uninfected at 7 days following administration of the indicated cumulative number of SHIV challenges. Statistical differences between groups were analyzed using a twosided log-rank test. Plasma levels of 10-1074 $\left(\mu \mathrm{g} \mathrm{ml}^{-1}\right)$ or 3BNC117 $\left(\mu \mathrm{g} \mathrm{ml}^{-1}\right)$ were determined via TZM-bl neutralization assays using 10-1074-sensitive pseudovirus X2088.c9 or 3BNC117-sensitive pseudovirus Q769.d22 for macaques that received both 10-1074 and 3BNC117 (f) or 3BNC117 alone (g). h Plasma concentrations of 10-1074 or 3BNC117 in individual macaques ( $N=5$ in Group-1, N=6 in Group-2) at the time of SHIV breakthrough (values from $\mathbf{f}$ (Group-1) or $\mathbf{g}$ (Group-2)). Solid lines denote group medians; dotted lines indicate the limits of detection for 10-1074 (black) or 3BNC117 (gray). Source data are provided in Supplementary Tables or a Source data file. 


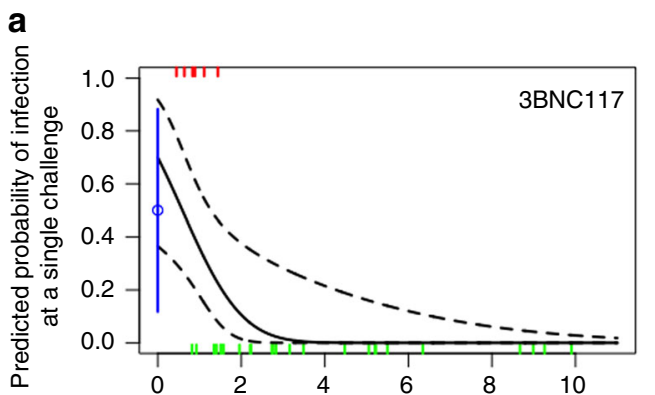

b

Model-based bNAb concentration $\left(\mu \mathrm{g} \mathrm{ml}^{-1}\right)$ at time of vaginal challenge

C

\begin{tabular}{ccc}
$\begin{array}{c}\text { 3BNC117 } \\
\left(\mu \mathrm{ml}^{-1}\right)\end{array}$ & $\begin{array}{c}\text { Infection probability per } \\
\text { vaginal SHIV challenge }\end{array}$ & $\begin{array}{c}\text { Estimated relative reduction } \\
\text { of infection probability } \\
\text { compared to controls }\end{array}$ \\
\hline 5.0 & $<0.001$ & $>0.99$ \\
3.0 & 0.016 & 0.97 \\
1.0 & 0.360 & 0.28
\end{tabular}

d

\begin{tabular}{ccc}
$\begin{array}{c}10-1074 \\
\left(\mu \mathrm{ml}^{-1}\right)\end{array}$ & $\begin{array}{l}\text { Infection probability per } \\
\text { vaginal SHIV challenge }\end{array}$ & $\begin{array}{c}\text { Estimated relative reduction } \\
\text { of infection probability } \\
\text { compared to controls }\end{array}$ \\
\hline 5.0 & $<0.001$ & $>0.99$ \\
3.0 & $<0.001$ & $>0.99$ \\
1.0 & 0.014 & 0.97
\end{tabular}

Fig. 4 Antibody concentration predicts the probability of infection following vaginal SHIV $\mathbf{A D B}_{\text {-EO }}$ challenge. Probit regression analysis was used to estimate the per-challenge probability of vaginal SHIV acquisition as a function of imputed 3 BNC117 concentrations in plasma among macaques that were administered 3BNC117 singly (a) or 10-1074 concentrations in plasma among animals that received 10-1074 in combination with 3BNC117 (b). The perchallenge probability of infection among untreated control animals was 0.50 and is denoted in blue. Tick marks represent SHIV challenge events that either

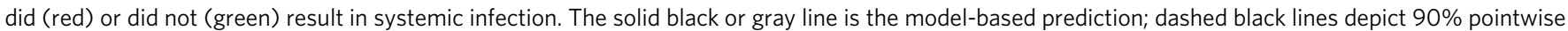
confidence intervals. Model-based estimates of SHIV infection probabilities following a single vaginal SHIV challenge at the indicated plasma concentrations of 3BNC117 (c) or 10-1074 (d).

Beginning 1 week following antibody injection, plasma levels of 10-1074 and 3BNC117 were measured among macaques that had received these two antibodies in combination. The maximum concentration of 10-1074 (mean $C_{\max } 10-1074=$ $35.7 \pm 9.6 \mu \mathrm{g} \mathrm{ml}^{-1}$ ) was 6 times as high as $3 \mathrm{BNC} 117$ (mean $\left.C_{\max } 3 \mathrm{BNC} 117=5.9 \pm 2.6 \mu \mathrm{g} \mathrm{ml}^{-1}\right)(P=0.0022$, paired $t$-test; Fig. 3f), and both maxima were observed at the earliest timepoint (7 days) that was sampled following antibody injection. Among macaques that received only $3 \mathrm{BNC} 117$, the mean maximum plasma level of 3BNC117 (mean $C_{\max } 3 \mathrm{BNC117}$ $=7.0 \pm 3.2 \mu \mathrm{g} \mathrm{ml}^{-1}$; Fig. $3 \mathrm{~g}$ ) also was observed at the earliest timepoint sampled following antibody injection (7 days post injection) and was similar in magnitude to that observed for 3BNC117 among animals in the combination treatment group (Fig. 3f; Supplementary Tables 2, 3). Thus, no adverse interaction by $10-1074$ on $3 \mathrm{BNC} 117$ pharmacokinetics was observed in vivo.

In macaques that were administered 3BNC117 singly, the median plasma 3 BNC117 concentration at the time of SHIV breakthrough was $0.50 \mu \mathrm{g} \mathrm{ml}^{-1} \quad\left(\right.$ range $=0.24-0.80 \mu \mathrm{g} \mathrm{ml}^{-1}$ ) (Fig. 3h). In contrast, at the time of SHIV breakthrough, macaques that had been administered both bNAbs exhibited a median plasma concentration for $10-1074$ of $0.10 \mu \mathrm{g} \mathrm{ml}^{-1}$ (range $=0.10-0.30 \mu \mathrm{g} \mathrm{ml}^{-1}$ ), but undetectable levels of 3BNC117 (Fig. 3h). Moreover, among all macaques in the combination $\mathrm{bNAb}$ group, 3BNC117 levels were at, or had been below, the level of detection $\left(0.24 \mu \mathrm{g} \mathrm{ml}^{-1}\right)$ for a median of 4 weeks (range $=3-8$ weeks) prior to SHIV breakthrough. This argues that the more durable protection observed in the combination antibody group was due to $10-1074$, which persisted relatively longer than $3 \mathrm{BNC} 117$. As such, these macaques were protected through a period of effective 10-1074 monotherapy. This allowed us to estimate levels of 10-1074 that were protective against vaginal SHIV $_{\text {AD8-EO }}$ infection, and enabled a comparison of correlates of protection for 10-1074 between the penile and vaginal challenge models.

We first used probit regression to estimate the per-challenge probability of SHIV infection as a function of imputed bNAb levels in plasma among animals in the vaginal challenge cohorts (Fig. 4). Among macaques that received 3BNC117 singly, this model estimated infection probabilities of $<0.001,0.016$, or 0.36 at $3 \mathrm{BNC} 117$ concentrations of $5.0,3.0$, or $1.0 \mu \mathrm{g} \mathrm{ml}^{-1}$, respectively. These estimates represent $>100-, 31-$, and 1.4-fold reductions in infection probability, respectively, from that of no-antibody controls whose per-challenge infection probability was 0.50 (95\% CI: 0.11-0.88). Bootstrap fitting of this model estimated a 0.01 probability of infection following a single vaginal SHIV challenge at a plasma 3 BNC117 concentration of $3.22 \mu \mathrm{g} \mathrm{ml}^{-1}$ (95\% CI: $1.62,4.69 \mu \mathrm{g} \mathrm{ml}^{-1}$ ).

Among macaques in the combination treatment group, probit regression estimated per-challenge infection probabilities of $<0.001,<0.001$, or 0.014 at $10-1074$ concentrations of $5.0,3.0$, or $1.0 \mu \mathrm{g} \mathrm{ml}^{-1}$, respectively. These estimates represent $>100-$, $>100-$, and 36-fold reductions as compared with untreated controls. Bootstrap fitting of the model showed a plasma 10-1074 concentration of $1.13 \mu \mathrm{g} \mathrm{ml}^{-1}$ (95\% CI: $0.51,1.53 \mu \mathrm{g}$ $\mathrm{ml}^{-1}$ ) predicted a 0.01 probability of infection following a single vaginal SHIV $\mathrm{AD}_{\mathrm{ADO}}$ challenge. Thus, among vaginally challenged macaques, the estimated plasma bNAb concentration corresponding to a 0.01 probability of infection was three times as high for 3BNC117 $\left[3.22 \mu \mathrm{g} \mathrm{ml}^{-1}\right.$ (95\% CI: $\left.\left.1.62,4.69 \mu \mathrm{g} \mathrm{ml}^{-1}\right)\right]$ than 10-1074 [1.13 $\mathrm{g} \mathrm{ml}^{-1}$ (95\% CI: $\left.\left.0.51,1.53 \mu \mathrm{g} \mathrm{ml}^{-1}\right)\right]$, which reflects the relatively lower neutralization potency of 3BNC117, as compared with 10-1074, against the challenge virus.

In contrast, comparison of the correlates of 10-1074-mediated protection between the penile and vaginal SHIV challenge models showed no significant difference-the estimated plasma 10-1074 concentration that corresponded to a 0.01 probability of SHIV $_{\text {SF162P3 }}$ infection via the penis $\left[2.28 \mu \mathrm{g} \mathrm{ml}^{-1}\right.$ (95\% CI: 1.27 , $\left.3.47 \mu \mathrm{g} \mathrm{ml}^{-1}\right)$ ] was not different from that against $\mathrm{SHIV}_{\mathrm{AD} 8-\mathrm{EO}}$ infection vaginally $\left[1.13 \mu \mathrm{g} \mathrm{ml}^{-1}\left(95 \% \mathrm{CI}: 0.51,1.53 \mu \mathrm{g} \mathrm{ml}^{-1}\right)\right]$.

\section{Discussion}

HIV infection of the penis has contributed substantially to the global HIV epidemic, as a majority of the $\sim 18$ million men living with HIV 
worldwide became infected through heterosexual transmission ${ }^{46}$. However, preclinical animal modeling to assess the efficacy of candidate HIV interventions against penile infection has lagged that targeting rectal or vaginal infection routes. Here, using a recently developed macaque model ${ }^{33}$, we provide a preclinical evaluation of bNAb-mediated protection against penile SHIV infection.

Our results demonstrated durable protection (up to 19 weeks) against penile $\mathrm{SHIV}_{\mathrm{SF} 162 \mathrm{P} 3}$ infection of macaques following a single subcutaneous injection of 10-1074. Probit regression modeling estimated that 10-1074 reduced the per-challenge penile infection risk by $>2$-logs, from 0.25 among untreated controls to $<0.001$ among macaques with a plasma $10-1074$ concentration of $5.0 \mu \mathrm{g} \mathrm{ml}^{-1}$. In phase-I studies among HIV uninfected people, 10-1074 concentrations $>5.0 \mu \mathrm{g} \mathrm{ml}^{-1}$ were observed for at least 8 weeks following either a single infusion of $10-1074$ at 3,10 , or $30 \mathrm{mg} \mathrm{kg}^{-1}$ or repeated infusions, once every 8 weeks, of 10-1074 in combination with $3 \mathrm{BNC} 117$ at $10 \mathrm{mg} \mathrm{kg}^{-117,20}$. If directly translatable, our results suggest that relatively infrequent dosing with 10-1074 might provide a high level of protection against penile HIV infection among men.

Our vaginal challenge study also provided information on correlates of bNAb-mediated protection. Among macaques administered 10-1074 and 3BNC117 in combination, protection was attributed to 10-1074 due to the observed difference in antibody pharmacokinetics following subcutaneous administration that resulted in a period of effective 10-1074 monotherapy preceding SHIV $_{\mathrm{AD} 8-\mathrm{EO}}$ infection. Probit regression modeling estimated that per-challenge vaginal infection risk was reduced by $>2$-logs, from 0.50 among untreated controls to $<0.001$ among macaques with a plasma $10-1074$ concentration of $3.0 \mu \mathrm{g} \mathrm{ml}^{-1}$ or greater.

A limitation in directly comparing the correlates of 10-1074mediated protection against penile or vaginal infection in this study is that the underlying animal models utilized different challenge virus strains and doses. Here, our penile and vaginal challenge models used SHIVs that are similarly susceptible to neutralization by $10-1074$, with $\mathrm{IC}_{80}$ values of 0.20 or $0.25 \mu \mathrm{g} \mathrm{ml}^{-1}$ against the replication competent challenge virus stock of $\mathrm{SHIV}_{\mathrm{SF} 162 \mathrm{P} 3}$ or SHIV $_{\mathrm{AD} 8-\mathrm{EO}}$, respectively. Regarding challenge virus dose selection, model feasibility necessitates that each SHIV exposure presents a relatively higher risk of infection to macaques, than does exposure of men or women to an HIV-infected source, for which the per-exposure infection rates are low (insertive vaginal -0.0004 , insertive rectal -0.0011 , receptive vaginal -0.0008$)^{1}$. For these macaque studies, challenge virus doses were selected empirically to normalize the number of challenges required to infect animals via penile or vaginal routes and resulted in a median of 2 or 2.5 challenges, respectively. Importantly, the bootstrapped estimate of the per-challenge probability of infection among untreated control animals in the penile $\mathrm{SHIV}_{\mathrm{SF} 162 \mathrm{P} 3}$ model [0.25 (95\% CI: 0.13-0.41)] was not significantly different from that among vaginal $\mathrm{SHIV}_{\mathrm{AD} 8-\mathrm{EO}}$ controls $[0.50$ (95\% CI: 0.11-0.88)]. Thus, macaques were challenged with SHIVs, via a penile or vaginal route, under model conditions of comparable stringency. These model-specific differences notwithstanding, we found no significant difference between the plasma 10-1074 concentration that reduced per-exposure infection risk to 0.01 against penile $\mathrm{SHIV}_{\mathrm{SF162 \textrm {P } 3}}$ or vaginal $\mathrm{SHIV}_{\mathrm{AD} 8-\mathrm{EO}}$ challenges, and risk reduction was greater than 100-fold for both groups when plasma $10-1074$ concentrations were $5.0 \mu \mathrm{g} \mathrm{ml}^{-1}$ or higher.

Likewise, these correlates of protection against penile or vaginal infection compare favorably with those reported for 10-1074mediated protection against repeated rectal $\mathrm{SHIV}_{\mathrm{AD} 8-\mathrm{EO}}$ challenges among macaques ${ }^{23}$. For example, at a plasma $10-1074$ concentration of $1.0 \mu \mathrm{g} \mathrm{ml}^{-1}, 63$ or $84 \%$ reductions in per-challenge infection risk were observed among macaques challenged repeatedly with $\mathrm{SHIV}_{\mathrm{SF162P3}}$ via the penis or SHIV $\mathrm{AD}$-EO rectally ${ }^{23}$. These findings are consistent with an earlier study showing passively administered
bNAb PGT126 protected similarly against a single high-dose challenge with $\mathrm{SHIV}_{\mathrm{SF} 162 \mathrm{P} 3}$ via rectal or vaginal routes ${ }^{47}$.

The site(s) and mechanism(s) by which passively administered bNAbs confer protection against penile or other mucosal routes of infection are not known fully. Transudation or active (FcRnmediated) transport of bNAbs from circulation to mucosal tissues or secretions would position these effector molecules at the point of virus entry, where their neutralization or Fc-related antiviral functions may be expected to have greatest impact to prevent either initial infection or local amplification and systemic spread of a nascent infection ${ }^{48-51}$. Observation that the plasma correlates of 10-1074-mediated protection were not different against penile or vaginal infection may reflect similar bNAb pharmacokinetics among these anatomic compartments or may reflect common antiviral effects occurring elsewhere than the mucosal portal of virus entry. Thus, a second limitation of this study was the lack of mucosal sampling to determine bNAb pharmacokinetics in penile tissues or secretions. However, because our primary study objective was protective efficacy, we chose not to collect penile samples from animals being challenged, out of concern that the collection procedures themselves would alter susceptibility to SHIV acquisition. Future studies to characterize the pharmacokinetics of bNAbs in penile tissues or secretions, following passive administration, should be informative in this regard.

We note that our penile infection model is a model of HIV infection among uncircumcised men. Because $60-70 \%$ of adult men globally are not circumcised ${ }^{52,53}$, this model has broad public health relevance. However, penile tissues other than the foreskin are relevant for HIV infection, as evidenced by the incomplete protection that circumcision confers against penile infection $^{54-56}$, as well as the wide distribution of HIV target cells among other penile tissues, including the urethra and glans ${ }^{25,57,58}$. Although our current challenge model simultaneously exposes multiple penile tissues to SHIV, it is of interest to determine whether removal of the foreskin via circumcision would alter the capacity of bNAbs to protect remaining penile tissues from infection. A final model limitation is that the nontraumatic technique used for penile challenges with SHIV does not account for any trauma that might occur to the penis during sexual intercourse.

In summary, we have utilized a recently developed macaque model of penile HIV infection to demonstrate protective efficacy of a passively administered bNAb against this route of infection. We determined plasma 10-1074 concentrations as correlates of protection against penile or vaginal SHIV acquisition and compared these to values reported to protect against rectal SHIV infection. Despite model-specific differences, the findings from these macaque studies show that large reductions in infection probability for all major routes of HIV acquisition could be demonstrated at clinically relevant levels of circulating bNAbs. Such an overall indication from macaque modeling should facilitate dose selection of bNAbs for clinical advancement of immunoprophylaxis against HIV.

\section{Methods}

Macaques. Thirty adult rhesus macaques (Macaca mulatta) were used to perform the penile or vaginal SHIV challenge studies, respectively. All animals were housed the Centers for Disease Control and Prevention (CDC; Atlanta, GA) in accordance with the Guide for the Care and Use of Laboratory Animals (8th edition) in an AAALACaccredited facility, according to institutional standard operating procedures. For

housing, macaques were maintained in cages that met or exceeded the minimum size requirements as stipulated in the Guide. Animals were provided enrichments that included objects to manipulate, assortments of fresh fruits and vegetables, suitable feeding methods (foraging and task-oriented), and humane interactions with caregivers and research staff. Prior to the initiation of virus challenges, compatible macaques were pair-housed to the extent possible. Animal studies were approved by the CDC Institutional Animal Care and Use Committee (IACUC, protocol 2804GARMONC). To 
minimize animal discomfort or suffering, all biomedical procedures were performed on animals under ketamine $\left(10 \mathrm{mg} \mathrm{kg}^{-1}\right)$ or Telazol $\left(2-6 \mathrm{mg} \mathrm{kg}^{-1}\right)$ anesthesia.

Challenge virus stocks. Preparation and characterization of the cell-free SHIV $_{\text {SF162P3 }}$ stock used to perform penile challenges has been described ${ }^{33}$-the undiluted stock had a titer of $2430 \mathrm{TCID}_{50} \mathrm{ml}^{-1}$, determined on whole, unstimulated primary rhesus PBMCs. A cell-free stock of SHIV $_{\mathrm{AD} 8-\mathrm{EO}}$, which was used to perform vaginal challenges, was prepared as follows. Infectious virus was obtained in supernatants of $293 \mathrm{~T}$ cell cultures at $48 \mathrm{~h}$ following FuGene 6 (Promega)-mediated transfection of plasmid pSHIV AD8-EO (kindly provided by Malcolm Martin, NIAID). Virus in 293T culture supernatants was amplified in rhesus macaque PBMCs following in vitro depletion of CD8 ${ }^{+}$cells (Dynabeads CD8, ThermoFisher) and stimulation with Concanavalin-A (Sigma-Aldrich). Supernatants were clarified via centrifugation, aliquoted and stored in the vapor phase of liquid nitrogen. The undiluted SHIV ${ }_{\text {ADs-EO }}$ stock had a titer of 3600 $\mathrm{TCID}_{50} \mathrm{ml}^{-1}$, determined on whole, unstimulated primary rhesus PBMCs.

Antibodies and passive immunization. Monoclonal antibodies 10-1074 and 3BNC117 were produced in the laboratory of M.C.N. and formulated individually for injection at concentrations ranging between 49.5 and $53 \mathrm{mg} \mathrm{ml}^{-1}$ in $5 \mathrm{mM}$ acetate, $280 \mathrm{mM}$ trehalose, $0.05 \%$ Tween 20 (pH 5.2) or $10 \mathrm{mM}$ L-histidine, $280 \mathrm{mM}$ trehalose, $0.05 \%$ Tween 20 ( $\mathrm{pH} 5.5$ ), for 10-1074 and 3BNC117, respectively ${ }^{17,18}$. Antibodies were administered via subcutaneous injection in macaques at $10 \mathrm{mg} \mathrm{kg}^{-1}$ in the upper back (3BNC117 on the left side, 10-1074 on the right side) via $22 \mathrm{G} 1$ needle; injection volumes were $<2 \mathrm{ml}$ per injection site.

Virus challenges. SHIV challenges were performed once weekly, via penile or vaginal routes, until systemic infection was confirmed by detection of vRNA in plasma. Penile SHIV challenges were performed by administering 200 TCID $_{50}$ into the prepuce (foreskin) pouch and $16 \mathrm{TCID}_{50}$ virus into the distal urethra. Urethral inoculations were performed using a 'no-contact' technique in which the lobes of the glans were manually flared to expose the navicular fossa and a $20 \mu$ inoculum volume was expelled from a micropipettor tip positioned 1-5 $\mathrm{mm}$ above the center of the exposed urethral opening ${ }^{33}$. This technique avoids all contact of the pipet tip with urethral tissue and precludes inadvertent abrasion of the urethral epithelium. SHIV $_{\text {SF162P3 }}$ was used for penile challenges as it is sensitive to neutralization by bNAb $10-1074$, and the penile model had been optimized with SHIV $\mathrm{SF162P3}_{3}$. For vaginal SHIV challenge, macaques were nontraumatically administered 300 $\mathrm{TCID}_{50}$ virus, in a $1 \mathrm{ml}$ volume, via an inserted pediatric nasogastric feeding tube of adjusted length. SHIV $\mathrm{ADB}_{\mathrm{ADO}}$ was selected for vaginal studies because we wanted to evaluate two bNAbs-3BNC117 and 10-1074, both of which neutralize this virus.

Viral load assay. Viral RNA in plasma was quantified using a real-time reverse transcription PCR assay to detect the SIV gag gen ${ }^{59}$. SHIV virions were concentrated from $1 \mathrm{ml}$ plasma samples via ultracentrifugation $\left(100,000 \mathrm{~g}\right.$ for $30 \mathrm{~min}$ at $\left.4{ }^{\circ} \mathrm{C}\right)$ and subjected to RNA extraction using NucliSens reagents (Biomerieux). One-step RTPCR was performed using the SuperScript III Platinum One-Step qRT-PCR Kit with ROX (ThermoFisher) with forward primer (SIVplf1) 5'-GCCAACAGGCTCAGAAA ATTTAA- $3^{\prime}$, reverse primer (SIVp1r1) 5'-TCCTCAGTGTGTTTCACTTTCTCTT C- $3^{\prime}$ and probe $5^{\prime}$-HEX-AGCCTTTATAATACTGTCTGCGTCATCTGGTGC-BH Q1-3'. The limit of detection was 60 viral RNA copies per $\mathrm{ml}$.

Determination of 10-1074 and 3BNC117 concentrations. Concentrations of $10-1074$ and 3BNC117 in plasma were determined using TZM-bl neutralization assays using pseudovirus X2088.c9, which is sensitive to neutralization by $10-1074$ but resistant to $3 \mathrm{BNC117}$, or Q769.d22, which is sensitive to 3BNC117, but resistant to $10-1074^{20}$. Inhibitory dilution $50 \%$ ( $\mathrm{ID}_{50}$ ) titers of pseudovirus neutralization by plasma samples were determined via five-parameter curve fitting. Plasma antibody concentration was calculated by multiplying a sample's $\mathrm{ID}_{50}$ titer by the inhibitory concentration $50 \%$ value from $10-1074$ or $3 \mathrm{BNC} 117$ reference lots, which were assayed in parallel. Plasma samples were measured against murine leukemia virus to detect any nonspecific activity. All samples were assayed in duplicate. Induction of endogenous (rhesus) antibody responses against SHIV Env following repeated SHIV challenges, but prior to systemic infection, could confound use of the TZM-bl assay to determine plasma bNAb concentrations. SHIV $_{\text {SF162P3 }}$ Env-specific antibody responses were not detected prior to SHIV breakthrough among macaques that received 10-1074 and were challenged repeatedly with SHIV $\mathrm{SF162 \textrm {P } 3}$ via the penis (Supplementary Fig. 4).

Detection of antidrug antibody (ADA) responses. ADA responses were evaluated in macaque plasma samples via ELISA. ELISA plates (96-well, high binding plates (Corning)) were coated overnight with $1 \mu \mathrm{g} 10-1074$ per well, then blocked with phosphate buffered saline containing $1 \%$ bovine serum albumin (PBS- $1 \%$ BSA). Plasma samples were diluted 1:50 in PBS-1\%BSA, added to the plates $(100 \mu \mathrm{l}$ per well) and incubated for $1.5-2.0 \mathrm{~h}$ at $37^{\circ} \mathrm{C}$. Plates were washed and incubated with mouse antirhesus IgG heavy chain-horseradish peroxidase (HRP) preadsorbed (Abcam) for $30 \mathrm{~min}$ at $37^{\circ} \mathrm{C}$. Plates were washed and assayed for HRP activity by the addition of tetramethylbenzidine (1-Step Ultra TMB,
ThermoFisher). Color development was arrested via the addition of sulfuric acid to a final concentration of $1 \mathrm{~N}$ and absorbance at $450 \mathrm{~nm}$ was measured using a BioTek ELx808 plate reader.

Statistical analysis. Statistical comparisons between groups using $t$-, Mann-Whitney, or log-rank tests were conducted using Prism 7.0 (GraphPad Software, Inc., San Diego, CA). Probit modeling and bootstrap analyses were conducted in $\mathrm{R}$ version 3.4.4.

Reporting summary. Further information on research design is available in the Nature Research Reporting Summary linked to this article.

\section{Data availability}

All relevant data are available from the authors. The source data underlying Figs. 1e, $3 \mathrm{f}$ and $3 \mathrm{~g}$ are included in Supplementary Tables 1,2, and 3, respectively. The source data underlying Figs. 1b, c, f, 3b, c, d, h are provided as a Source Data File.

Received: 21 October 2019; Accepted: 1 June 2020;

Published online: 24 June 2020

\section{References}

1. Patel, P. et al. Estimating per-act HIV transmission risk: a systematic review AIDS 28, 1509-1519 (2014).

2. Giannou, F. K. et al. Condom effectiveness in reducing heterosexual HIV transmission: a systematic review and meta-analysis of studies on HIV serodiscordant couples. Expert Rev. Pharmacoecon. Outcomes Res. 16, 489-499 (2016).

3. Baeten, J. M. et al. Antiretroviral prophylaxis for HIV prevention in heterosexual men and women. N. Engl. J. Med. 367, 399-410 (2012).

4. Thigpen, M. C. et al. Antiretroviral preexposure prophylaxis for heterosexual HIV transmission in Botswana. N. Engl. J. Med. 367, 423-434 (2012).

5. Siegfried N., Muller M., Deeks J. J., Volmink J. Male circumcision for prevention of heterosexual acquisition of HIV in men. Cochrane Database Syst. Rev. 2, Cd003362 (2009).

6. Grant, R. M. et al. Preexposure chemoprophylaxis for HIV prevention in men who have sex with men. The. N. Engl. J. Med. 363, 2587-2599 (2010).

7. McCormack, S. et al. Pre-exposure prophylaxis to prevent the acquisition of HIV-1 infection (PROUD): effectiveness results from the pilot phase of a pragmatic open-label randomised trial. Lancet 387, 53-60 (2016).

8. Molina, J. M. et al. On-demand preexposure prophylaxis in men at high risk for HIV-1 infection. N. Engl. J. Med. 373, 2237-2246 (2015).

9. Chou, R. et al. U.S. Preventive Services Task Force Evidence Syntheses, formerly Systematic Evidence Reviews. In: Pre-Exposure Prophylaxis for the Prevention of HIV Infection: A Systematic Review for the U.S.

Preventive Services Task Force (Agency for Healthcare Research and Quality, US, 2019).

10. Gilbert, P. B. et al. Basis and statistical design of the passive HIV-1 antibody mediated prevention (AMP) test-of-concept efficacy trials. Stat. Commun. Infect. Dis. 9, https://doi.org/10.1515/scid-2016-0001 (2017).

11. Sullivan, P. S., Salazar, L., Buchbinder, S. \& Sanchez, T. H. Estimating the proportion of HIV transmissions from main sex partners among men who have sex with men in five US cities. AIDS 23, 1153-1162 (2009).

12. Caskey, M., Klein, F. \& Nussenzweig, M. C. Broadly neutralizing anti-HIV-1 monoclonal antibodies in the clinic. Nat. Med. 25, 547-553 (2019).

13. Sok, D. \& Burton, D. R. Recent progress in broadly neutralizing antibodies to HIV. Nat. Immunol. 19, 1179-1188 (2018).

14. Grobben, M., Stuart, R. A. \& van Gils, M. J. The potential of engineered antibodies for HIV-1 therapy and cure. Curr. Opin. Virol. 38, 70-80 (2019).

15. Scheid, J. F. et al. Sequence and structural convergence of broad and potent HIV antibodies that mimic CD4 binding. Science 333, 1633-1637 (2011).

16. Mouquet, $\mathrm{H}$. et al. Complex-type $\mathrm{N}$-glycan recognition by potent broadly neutralizing HIV antibodies. Proc. Natl. Acad. Sci. USA 109, E3268-E3277 (2012).

17. Caskey, M. et al. Antibody 10-1074 suppresses viremia in HIV-1-infected individuals. Nat. Med. 23, 185-191 (2017)

18. Caskey, M. et al. Viraemia suppressed in HIV-1-infected humans by broadly neutralizing antibody 3BNC117. Nature 522, 487-491 (2015).

19. Scheid, J. F. et al. HIV-1 antibody 3BNC117 suppresses viral rebound in humans during treatment interruption. Nature 535, 556-560 (2016).

20. Cohen, Y. Z. et al. Safety, pharmacokinetics, and immunogenicity of the combination of the broadly neutralizing anti-HIV-1 antibodies 3BNC117 and 10-1074 in healthy adults: a randomized, phase 1 study. PloS One 14, e0219142 (2019). 
21. Hessell, A. J. \& Haigwood, N. L. Animal models in HIV-1 protection and therapy. Curr. Opin. HIV AIDS 10, 170-176 (2015).

22. Anderson, D. J. et al. Systemic and topical use of monoclonal antibodies to prevent the sexual transmission of HIV. AIDS 31, 1505-1517 (2017).

23. Gautam, R. et al. A single injection of anti-HIV-1 antibodies protects against repeated SHIV challenges. Nature 533, 105-109 (2016).

24. Shingai, M. et al. Passive transfer of modest titers of potent and broadly neutralizing anti-HIV monoclonal antibodies block SHIV infection in macaques. J. Exp. Med. 211, 2061-2074 (2014).

25. Anderson, D., Politch, J. A. \& Pudney, J. HIV infection and immune defense of the penis. Am. J. Reprod. Immunol. 65, 220-229 (2011).

26. Anderson, D. J., Marathe, J. \& Pudney, J. The structure of the human vaginal stratum corneum and its role in immune defense. Am. J. Reprod. Immunol. 71, 618-623 (2014).

27. Hladik, F. \& McElrath, M. J. Setting the stage: host invasion by HIV. Nat. Rev. Immunol. 8, 447-457 (2008)

28. Abdool Karim, S. S., Baxter, C., Passmore, J. S., McKinnon, L. R. \& Williams, B. L. The genital tract and rectal microbiomes: their role in HIV susceptibility and prevention in women. J. Int. AIDS Soc. 22, e25300 (2019).

29. Salas, J. T. \& Chang, T. L. Microbiome in human immunodeficiency virus infection. Clin. Lab. Med. 34, 733-745 (2014).

30. Carias, A. M. et al. Defining the interaction of HIV-1 with the mucosal barriers of the female reproductive tract. J. Virol. 87, 11388-11400 (2013).

31. Dinh, M. H., Okocha, E. A., Koons, A., Veazey, R. S. \& Hope, T. J. Expression of structural proteins in human female and male genital epithelia and implications for sexually transmitted infections. Biol. Reprod. 86, 32 (2012).

32. Dinh, M. H. et al. Visualization of HIV-1 interactions with penile and foreskin epithelia: clues for female-to-male HIV transmission. PLoS Pathog. 11, e1004729 (2015).

33. Garber, D. A. et al. Development of a repeat-exposure penile SHIV infection model in macaques to evaluate biomedical preventions against HIV. PloS one 13, e0194837 (2018)

34. Bbosa, N., Kaleebu, P. \& Ssemwanga, D. HIV subtype diversity worldwide. Curr. Opin. HIV AIDS 14, 153-160 (2019).

35. Wagh, K. et al. Optimal combinations of broadly neutralizing antibodies for prevention and treatment of HIV-1 Clade C infection. PLoS Pathog. 12, e1005520 (2016).

36. Wagh, K. et al. Potential of conventional \& bispecific broadly neutralizing antibodies for prevention of HIV-1 subtype A, C \& D infections. PLoS Pathog. 14, e1006860 (2018)

37. Doria-Rose, N. A. et al. HIV-1 neutralization coverage is improved by combining monoclonal antibodies that target independent epitopes. J. Virol. 86, 3393-3397 (2012).

38. Goo, L., Jalalian-Lechak, Z., Richardson, B. A. \& Overbaugh, J. A combination of broadly neutralizing HIV-1 monoclonal antibodies targeting distinct epitopes effectively neutralizes variants found in early infection. J. Virol. 86, 10857-10861 (2012).

39. Kong, R. et al. Improving neutralization potency and breadth by combining broadly reactive HIV-1 antibodies targeting major neutralization epitopes. $J$. Virol. 89, 2659-2671 (2015).

40. Julg, B. et al. Protection against a mixed SHIV challenge by a broadly neutralizing antibody cocktail. Sci. Transl. Med. 9, https://doi.org/10.1126/ scitranslmed.aao4235 (2017).

41. Seaman, M. S. et al. Optimization and qualification of a functional anti-drug antibody assay for HIV-1 bNAbs. J. Immunolog. Methods 479, 112736-112746 (2020).

42. Bharadwaj et al. Implementation of a three-tiered approach to identify and characterize anti-drug antibodies raised against HIV-specific broadly neutralizing antibodies. J. Immunolog. Methods 479, 112764-112775 (2020).

43. Hild-Petito, S., Veazey, R. S., Larner, J. M., Reel, J. R. \& Blye, R. P. Effects of two progestin-only contraceptives, Depo-Provera and Norplant-II, on the vaginal epithelium of rhesus monkeys. AIDS Res. Hum. Retrovir. 14, S125-S130 (1998).

44. Marx, P. A. et al. Progesterone implants enhance SIV vaginal transmission and early virus load. Nat. Med. 2, 1084-1089 (1996).

45. Veazey, R. S. et al. Prevention of virus transmission to macaque monkeys by a vaginally applied monoclonal antibody to HIV-1 gp120. Nat. Med. 9, 343-346 (2003).

46. UNAIDS. UNAIDS Data 2019. 16-18 (Joint United Nations Programme on HIV/AIDS, Geneva, Switzerland, 2019).

47. Moldt, B. et al. Neutralizing antibody affords comparable protection against vaginal and rectal simian/human immunodeficiency virus challenge in macaques. AIDS 30, 1543-1551 (2016).

48. Haase, A. T. Perils at mucosal front lines for HIV and SIV and their hosts. Nat. Rev. Immunol. 5, 783-792 (2005).

49. Liu, J. et al. Antibody-mediated protection against SHIV challenge includes systemic clearance of distal virus. Science 353, 1045-1049 (2016).
50. Ko, S. Y. et al. Enhanced neonatal Fc receptor function improves protection against primate SHIV infection. Nature 514, 642-645 (2014).

51. Lemos, M. P. et al. In men at risk of HIV infection, IgM, IgG1, IgG3, and IgA reach the human foreskin epidermis. Mucosal Immunol. 9, 798-808 (2016).

52. Male Circumcision: Global Trends and Determinants of Prevalence, Safety and Acceptability (World Health Organization, 2007) https://apps.who.int/iris/ bitstream/handle/10665/43749/9789241596169_enf.pdf; jsessionid $=0410849 \mathrm{DFA}$ 1EE6E3E7877F0D06B0F820? sequence $=1$.

53. Morris, B. J. et al. Estimation of country-specific and global prevalence of male circumcision. Popul. Health Metr. 14, 4 (2016).

54. Auvert, B. et al. Randomized, controlled intervention trial of male circumcision for reduction of HIV infection risk: the ANRS 1265 Trial. PLoS Med. 2, e298 (2005)

55. Bailey, R. C. et al. Male circumcision for HIV prevention in young men in Kisumu, Kenya: a randomised controlled trial. Lancet 369, 643-656 (2007).

56. Gray, R. H. et al. Male circumcision for HIV prevention in men in Rakai, Uganda: a randomised trial. Lancet 369, 657-666 (2007).

57. McCoombe, S. G. \& Short, R. V. Potential HIV-1 target cells in the human penis. AIDS 20, 1491-1495 (2006)

58. Fischetti, L., Barry, S. M., Hope, T. J. \& Shattock, R. J. HIV-1 infection of human penile explant tissue and protection by candidate microbicides. AIDS 23, 319-328 (2009).

59. Subbarao, S. et al. Chemoprophylaxis with tenofovir disoproxil fumarate provided partial protection against infection with simian human immunodeficiency virus in macaques given multiple virus challenges. J. Infect. Dis. 194, 904-911 (2006).

\section{Acknowledgements}

Funding for this study was from CDC intramural funds. The findings and conclusions in this paper are those of the authors and do not necessarily represent the official position of the Centers for Disease Control and Prevention or National Institutes of Health.

\section{Author contributions}

D.A.G., J.McN, M.C.N, and W.H. developed the concept and designed the experiments D.R.A., P.G., J.M., and K.K. performed experiments or animal procedures; A.G. generated bNAbs used in this study; M.S.S. and T.S. determined bNAb concentrations; M.N performed probit modeling and bootsrap analyses; D.A.G wrote the paper; All authors edited or reviewed the paper.

\section{Competing interests}

There are patents on 3BNC117 (US Provisional Application No. 61/715,642) and 10-1074 (US Provisional Application No. 61/486,960) on which MCN is an inventor. 10-1074 and 3BNC117 are included in licensing agreements between The Rockefeller University and Gilead Sciences. The authors declare no further competing interests.

\section{Additional information}

Supplementary information is available for this paper at https://doi.org/10.1038/s41467 020-16928-9.

Correspondence and requests for materials should be addressed to D.A.G.

Peer review information Nature Communications thanks Ian McGowan and the other anonymous, reviewer(s) for their contribution to the peer review of this work.

Reprints and permission information is available at http://www.nature.com/reprints

Publisher's note Springer Nature remains neutral with regard to jurisdictional claims in published maps and institutional affiliations.

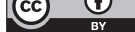

Open Access This article is licensed under a Creative Commons Attribution 4.0 International License, which permits use, sharing, adaptation, distribution and reproduction in any medium or format, as long as you give appropriate credit to the original author(s) and the source, provide a link to the Creative Commons license, and indicate if changes were made. The images or other third party material in this article are included in the article's Creative Commons license, unless indicated otherwise in a credit line to the material. If material is not included in the article's Creative Commons license and your intended use is not permitted by statutory regulation or exceeds the permitted use, you will need to obtain permission directly from the copyright holder. To view a copy of this license, visit http://creativecommons.org/ licenses/by/4.0/.

This is a U.S. government work and not under copyright protection in the U.S.; foreign copyright protection may apply 2020 